\title{
On the Integrality of Somos 4 Sequences
}

\author{
Betül GEZER iD \\ Bursa Uludağ Üniversitesi, Fen Edebiyat Fakültesi Matematik Bölümü, Bursa, Türkiye \\ Geliş / Received: 13/04/2018, Kabul / Accepted: 18/03/2019

\begin{abstract}
In this paper, it is shown that if $\left(h_{n}\right)$ is a Somos 4 sequence associated to an elliptic curve then each $h_{n}$ can be expressed as elements of the ring $R=\mathbb{Z}\left[a_{1}, a_{2}, a_{3}, a_{4}, x, y, h_{-1}{ }^{ \pm 1}, h_{0}\right]$. In particular, if $h_{-1}= \pm 1$, then the Somos 4 sequence consist entirely of integers for $n \geq 0$. Also the general term of the Somos 4 sequence is given which satisfies a binary recurrence relation.
\end{abstract}

Keywords: Somos 4 sequences, elliptic curves, linear sequences.

\section{Somos 4 Dizilerinin Tamsayılık Özelliği Üzerine}

Öz

Bu çalışmada, eğer $\left(h_{n}\right)$, bir eliptik eğri ile eşleşen bir Somos 4 dizisi ise her bir $h_{n}$ teriminin $R=\mathbb{Z}\left[a_{1}, a_{2}, a_{3}, a_{4}\right.$, $x, y, h_{-1}^{ \pm 1}, h_{0}$ ] halkasının bir elemanı olarak ifade edilebileceği gösterilmiştir. Özel olarak $n \geq 0$ için $h_{-1}= \pm 1$ ise Somos 4 dizisinin terimleri birer tamsayıdır. Üstelik bir ikili indirgeme bağıntısını gerçekleyen Somos 4 dizisinin genel terimi verilmiştir.

Anahtar Kelimeler: Somos 4 dizileri, eliptik eğriler, lineer diziler

\section{Introduction}

Linear recurrence sequences have been playing an important role in number theory. These sequences have many applications in approximation theory, cryptography and computer science. But there are also sequences which satisfy a bilinear recurrence relation. In the late $1980 \mathrm{~s}$, Micheal Somos discovered the sequence which begins

2. $1,1,1,1,1,1,3,5,9,23,75, \ldots$.

M. Somos see that the terms of the above sequence consist entirely of integers even though they are obtained from a rational recursion, see (Propp, 2018) for more details. Let $k \geq 4$ be an integer. A Somos $k$ sequence is a sequence $\left(h_{n}\right)$ which satisfies the recurrence relation

$$
h_{n} h_{n-k}=\sum_{i=1}^{\lfloor k / 2\rfloor} \tau_{i} h_{n-i} h_{n-k+i}
$$

where the coefficients $\tau_{i}$ and the initial values $h_{0}, \ldots, h_{k-1}$ are given integers. In particular, a
Somos $k$ sequence with all coefficients and initial values 1 is called $\operatorname{Somos}(k)$ sequence. Note that the recurrences defining Somos sequences indicate divisions by another term. It is clear that, these sequences turn out to have rational terms. Remarkably, the $\operatorname{Somos}(k)$ sequences have only integer terms for $k \leq 7$ but not for $k=8$ (Robinson, 1992; Gale, 1991). The question of when Somos sequences have only integer terms has received much attention in the literature (Fomin et al, 2002; Gale, 1991a and b; Hone and Swart, 2008; Malouf, 1992). A family of integer Somos sequence is also given by using the Tate normal form of an elliptic curve in Gezer et al (2016).

Robinson (1992) considered the properties of the Somos(4) sequence modulo prime powers. Swart (2003) extended his results to Somos 4 sequences and proved some of his conjectures.In this paper, we are interested in Somos 4 sequences which satisfy a recurrence relation 
$h_{n+2} h_{n-2}=\tau_{1} h_{n+1} h_{n-1}+\tau_{2} h_{n}^{2}$

where $\tau_{1}, \tau_{2}$ are given integers. These sequences are generalizations of elliptic divisibility sequences (EDSs). These sequences were first presented by M. Ward (1948b). Ward also showed that the terms of EDSs consist entirely of integers if the sequence begins $1, h_{2}, h_{3}, h_{2} c\left(h_{2}, h_{3}, c \in \mathbb{Z}\right)$. For more details see (Ward 1948a and b; Everest et al, 2003).Somos 4 sequences are also closely related to cluster algebras (Fomin and Zelevinsky, 2002), to integrable systems (Hone, 2006) and to continued fractions (van Der Poorten, 2005a and b). Fomin and Zelevinsky (2002) used the theory of cluster algebras to prove that all elements of the Somos 4 sequences are Laurent polynomials, i.e.,

$$
h_{n} \in \mathbb{Z}\left[\tau_{1}, \tau_{2}, h_{1}^{ \pm 1}, h_{2}^{ \pm 1}, h_{3}^{ \pm 1}, h_{4}^{ \pm 1}\right]
$$

for all $n \in \mathbb{Z}$.

Let $\left(h_{n}\right)$ be a Somos 4 sequence with coefficients $\tau_{1}, \tau_{2}$ and the initial values $h_{1}, h_{2}$, $h_{3}, h_{4}$, let $T$ be the quantity

$$
T=\frac{h_{1}^{2} h_{4}^{2}+\tau_{1}\left(h_{2}^{3} h_{4}+h_{1} h_{3}^{3}\right)+\tau_{1} h_{2}^{2} h_{3}^{2}}{h_{1} h_{2} h_{3} h_{4}}
$$

and let $I=\tau_{1}^{2}+\tau_{2} T$. Hone and Swart (2008) used the relation between Somos 4 sequences and elliptic curves and extended the known results on integrality of Somos 4 sequences. More precisely, Hone and Swart (2008) showed that the elements of the sequence $\left(h_{n}\right)$ are elements of the ring

$R=\mathbb{Z}\left[\tau_{1}, \tau_{2}, I, h_{1}^{ \pm 1}, h_{2}, h_{3}, h_{4}\right]$.

This gave a stronger Laurent phenomen for Somos 4 sequences. Moreover, Hone and Swart (2008) showed that if $\left(h_{n}\right)$ is a Somos 4 sequence with integer coefficients $\tau_{1}, \tau_{2}$ and non-zero integer initial values $h_{1}= \pm 1, h_{2}, h_{3}$, $h_{4}$, and if $\tau_{1} T \in \mathbb{Z}$, then $h_{n} \in \mathbb{Z}$ for all $n \geq 1$.

\section{Elliptic Curves and Somos Sequences}

Let $E$ denote an elliptic curve given by a Weierstrass equation
$E: y^{2}+a_{1} x y+a_{3} y=x^{3}+a_{2} x^{2}+a_{4} x+a_{6}$ (2.1)

with coefficients $a_{1}, \ldots, a_{6} \in \mathbb{Q}$. Let $E(\mathbb{Q})$ denote the set of rational points on $E$ together with the point at infinity $\boldsymbol{O}$. For more details on elliptic curves in general, see (Silverman, 2009; Silverman and Tate, 1992; Husemöller, 1987). In particular, Somos 4 sequences are quite interesting because of the close relation with elliptic curves. In fact, Somos 4 sequences with the first coefficient square can be expressed in terms of the $x$ coordinates of the points $\left(x_{n}, y_{n}\right)=Q+n P$ where $P=(0,0)$ and $Q=(x, y)$ is a suitable point on an elliptic curve.

The relation between an elliptic curve and a Somos 4 sequence is established independently by N. Elkies and N. Stephens (for more details see (Propp, 2018)). In (Swart, 2003), some unpublished works of N. Stephens were given, see also (Hone, 2005) for a different approach.

Let $P=(0,0)$ and $Q=(x, y)$ be non-singular integral points on $E$ such that $Q \neq \pm P, \boldsymbol{O}$ and $\left(x_{n}, y_{n}\right)=Q+n P \neq \boldsymbol{O}$ for all $n \in \mathbb{Z}$. The terms of Somos 4 sequence $\left(h_{n}\right)$ can be defined as follows: Let $h_{-1}$ and $h_{0}$ arbitrary non-zero integers and

$$
h_{n+1}=-\frac{x_{n} h_{n}^{2}}{h_{n-1}}
$$

for all $n \geq 0$ (see (Swart, 2003) for more details). The following result due to $\mathrm{N}$. Stephens.

Theorem 2.1. (Swart, 2003) Let $E$ denote an elliptic curve given by a Weierstrass equation (2.1) with integral coefficients $a_{1}, \ldots, a_{4}$. Let $P=(0,0)$ and $Q=(x, y)$ be non-singular integral points on $E$ such that $Q \neq \pm P, \boldsymbol{O}$ and $Q+n P \neq \boldsymbol{O}$ for all $n \in \mathbb{Z}$ and write $Q+n P=$ $\left(x_{n}, y_{n}\right)$. Then the coefficients $\tau_{1}, \tau_{2}$ and the initial values of the Somos 4 sequence associated to the elliptic curve $E$ given by

$$
\tau_{1}=a_{3}^{2}, \quad \tau_{2}=a_{4}\left(a_{4}+a_{1} a_{3}\right)-a_{3}^{2} a_{2}
$$

and $h_{-1}$ and $h_{0}$ arbitrary non-zero integers, 


$$
h_{1}=-\frac{x h_{0}^{2}}{h_{-1}}, \quad h_{2}=-\frac{\left(a_{4} x-a_{3} y\right) h_{0}^{3}}{h_{-1}^{2}} \text {. }
$$

Let $E$ be an elliptic curve given by equation (2.1) with integral coefficients and let $\left(h_{n}\right)$ be the Somos 4 sequence associated to $E$. In this paper we show that elements of the Somos 4 sequence $\left(h_{n}\right)$ associated to $E$ can be expressed as elements of the ring

$$
R=\mathbb{Z}\left[a_{1}, a_{2}, a_{3}, a_{4}, x, y, h_{1}^{ \pm 1}, h_{0}\right] .
$$

Thus we obtain that the Somos 4 sequence $\left(h_{n}\right)$ with $h_{-1}= \pm 1$ associated to $E$ consists entirely of integers for all $n \geq 0$. The proof of our theorem uses the relations in Theorem 2.1 and the elliptic equation (2.1), while in (Hone and Swart, 2008), the authors use the invariant (1.3) to characterize that a Somos 4 sequence $\left(h_{n}\right)$ associated to an elliptic curve. Hence they obtain the integrality properties for Somos 4 sequences by using the invariant (1.3), see (Hone and Swart, 2008).

Theorem 2.2. Let $E$ denote an elliptic curve given by a Weierstrass equation (2.1) with integral coefficients and let $P=(0,0)$ and $Q$ $=(x, y)$ be non-singular integral points on $E$ such that $Q \neq \pm P, \boldsymbol{O}$ and $Q+n P \neq \boldsymbol{O}$ for all $n$ $\in \mathbb{Z}$. Let $\left(h_{n}\right)$ denote a Somos 4 sequence with $h_{-1}= \pm 1$ associated to an elliptic curve $E$. Then the Somos 4 sequence $\left(h_{n}\right)$ consists entirely of integers for all $n \geq 0$.

\section{Proof of Theorem 2.2}

We first state a lemma to prove the main theorem.

Lemma 3.1. Let $E$ denote an elliptic curve given by equation (2.1) with integral coefficients and let $P=(0,0)$ and $Q=(x, y)$ be non-singular integral points on $E$ such that $Q \neq \pm P, \boldsymbol{O}$ and $Q+n P \neq \boldsymbol{O}$ for all $n \in \mathbb{Z}$. Let $\left(h_{n}\right)$ be a Somos 4 sequence associated to an elliptic curve $E$. Then each $h_{n}$ can be expressed as elements of the ring

$$
R=\mathbb{Z}\left[a_{1}, a_{2}, a_{3}, a_{4}, x, y, h_{1}^{ \pm 1}, h_{0}\right] .
$$

In particular, if $h_{-1}= \pm 1$, then the Somos 4 sequence consists entirely of integers for all $n \geq 0$

Proof. of Lemma 3.1 Let $E$ be an elliptic curve and let $P$ and $Q$ be points on $E$ as above and let $h_{-1}, h_{0}$ be arbitrary non-zero integers. Then the coefficients $\tau_{1}, \tau_{2}$ and the initial values $h_{1}$, $h_{2}$ of the Somos 4 sequences associated to $E$ are

$$
\tau_{1}=b_{6}=a_{3}^{2}, \quad \tau_{2}=-b_{8}=a_{4}\left(a_{4}+a_{1} a_{3}\right)-a_{2} a_{3}^{2}
$$

and

$$
h_{1}=-x h_{0}^{2} / h_{-1}, \quad h_{2}=-\left(a_{4} x-a_{3} y\right) h_{0}^{3} / h_{-1}^{2} .
$$

Using the relations (1.2) and (2.1) we obtain

$$
\begin{gathered}
h_{3}=\left(-b_{8} x^{2}-a_{4} b_{6} x+a_{3}^{3} y\right) h_{0}^{4} / h_{-1}^{3}, \\
h_{4}=\left(b_{8} b_{6} x^{3}+a_{4}\left(-a_{4} b_{8}+b_{6}^{2}\right) x^{2}-\left(a_{3}^{5}-2 a_{4} b_{8}\right) x y-b_{8} b_{6} y^{2}\right) h_{0}^{5} / h_{-1}^{4}, \\
h_{5}=\left(-a_{4}^{3}\left(a_{1} a_{3}+a_{4}\right)^{3} x^{3}+a_{3}^{2} a_{4}^{2}\left(5 a_{1} a_{3} a_{4}^{2}+2 a_{4} a_{1}^{2} a_{3}^{2}+a_{3}^{4}+3 a_{4}^{3}\right) x^{2}\right. \\
-a_{3}^{3} a_{4}\left(5 a_{4}^{3}+2 a_{3}^{4}+8 a_{1} a_{3} a_{4}^{2}+8 a_{4} a_{1}^{2} a_{3}^{2}\right) x y \\
\left.+a_{3}^{4}\left(a_{3}^{4}+a_{4} a_{1}^{2} a_{3}^{2}-3 a_{1} a_{3} a_{4}^{2}+2 a_{4}^{3}\right) y^{2}\right) h_{0}^{6} / h_{-1}^{5}
\end{gathered}
$$

where $b_{i}$ 's are the usual quantities for the elliptic curve $E$ and 


$$
h_{6}=\left(A x^{3}+B x^{2} y+C x^{2}+D x y+E y^{2}\right) h_{0}^{7} / h_{-1}^{6}
$$

where

$$
\begin{aligned}
& A=-a_{1}^{3} a_{3}^{3} a_{4}^{6}+a_{1} a_{2}^{4} a_{3}^{9}-3 a_{1} a_{3} a_{4}^{8}-3 a_{2}^{2} a_{3}^{8} a_{4}^{2}-8 a_{2}^{3} a_{3}^{6} a_{4}^{3}+3 a_{2}^{4} a_{3}^{8}-12 a_{1} a_{3}^{5} a_{4}^{5}+9 a_{2} a_{3}^{6} a_{4}^{4} a_{4} \\
& -9 a_{1}^{2} a_{3}^{6} a_{4}^{4}-3 a_{1}^{2} a_{3}^{2} a_{4}^{7}+6 a_{2}^{2} a_{3}^{4} a_{4}^{5}+2 a_{2} a_{3}^{10} a_{4}-2 a_{1} a_{3}^{9} a_{4}^{2}-2 a_{1}^{3} a_{3}^{7} a_{4}^{3}-a_{1}^{4} a_{2} a_{3}^{6} a_{4}^{3}+12 a_{1} a_{2} a_{3}^{7} a_{4}^{3} \\
& -4 a_{1} a_{2} a_{3}^{3} a_{4}^{6}-9 a_{1}^{2} a_{2} a_{3}^{4} a_{4}^{5}-6 a_{1}^{3} a_{2} a_{3}^{5} a_{4}^{4}-12 a_{1} a_{2}^{3} a_{3}^{7} a_{4}^{2}+18 a_{1} a_{2}^{2} a_{3}^{5} a_{4}^{4}+15 a_{1}^{2} a_{2}^{2} a_{3}^{6} a_{4}^{3} \\
& -3 a_{1}^{2} a_{2}^{3} a_{3}^{8} a_{4}+3 a_{1}^{2} a_{2} a_{3}^{8} a_{4}^{2}+3 a_{1}^{3} a_{2}^{2} a_{3}^{7} a_{4}^{2}-a_{4}^{9}-2 a_{3}^{8} a_{4}^{3}-5 a_{3}^{6} a_{4}^{3}-a_{2}^{3} a_{3}^{10} \\
& B=12 a_{1} a_{2}^{2} a_{3}^{6} a_{4}^{3}-18 a_{1} a_{2} a_{3}^{8} a_{4}^{2}-24 a_{1} a_{2} a_{3}^{4} a_{4}^{5}-27 a_{1}^{2} a_{2} a_{3}^{5} a_{4}^{4}-14 a_{1}^{3} a_{2} a_{3}^{6} a_{4}^{3}+9 a_{1}^{2} a_{2}^{2} a_{3}^{7} a_{4}^{2} \\
& -6 a_{1}^{2} a_{2} a_{3}^{9} a_{4}+3 a_{1}^{3} a_{2}^{2} a_{3}^{8} a_{4}-3 a_{1}^{4} a_{2} a_{3}^{7} a_{4}^{2}-a_{2}^{4} a_{3}^{9}+2 a_{3}^{9} a_{4}^{2}+6 a_{3}^{5} a_{4}^{5}+3 a_{3} a_{4}^{8}-2 a_{2} a_{3}^{11}-a_{1}^{2} a_{2}^{3} a_{3}^{9} \\
& +a_{1}^{5} a_{3}^{6} a_{4}^{3}+15 a_{1} a_{3}^{6} a_{4}^{4}-12 a_{2} a_{3}^{7} a_{4}^{3}+12 a_{1}^{2} a_{3}^{7} a_{4}^{3}+19 a_{1}^{2} a_{3}^{3}+15 a_{1}^{3} a_{3}^{4} a_{4}^{5}+6 a_{2}^{2} a_{3}^{9} a_{4}+6 a_{1}^{4} a_{3}^{5} a_{4}^{4} a_{4}^{6} \\
& +12 a_{1} a_{3}^{2} a_{4}^{7}+6 a_{2}^{2} a_{3}^{5} a_{4}^{4}-8 a_{2} a_{3}^{3} a_{4}^{6}+2 a_{1} a_{3}^{10} a_{4}+3 a_{1} a_{2}^{2} a_{3}^{10}+3 a_{1}^{3} a_{3}^{8} a_{4}^{2}, \\
& C=a_{1} a_{3}^{7} a_{4}^{4}-a_{2}^{2} a_{3}^{10} a_{4}-a_{1}^{4} a_{3}^{6} a_{4}^{4}-6 a_{1}^{3} a_{3}^{5} a_{4}^{5}+3 a_{2}^{3} a_{3}^{8} a_{4}^{2}-10 a_{1} a_{3}^{3} a_{4}^{7}+9 a_{2} a_{3}^{4} a_{4}^{6}-12 a_{1}^{2} a_{3}^{4} a_{4}^{6} \\
& -9 a_{2}^{2} a_{3}^{6} a_{4}^{4}+a_{1} a_{2}^{3} a_{3}^{9} a_{4}+a_{1} a_{2} a_{3}^{9} a_{4}^{2}+21 a_{1} a_{2} a_{3}^{5} a_{4}^{5}-12 a_{1} a_{2}^{2} a_{3}^{7} a_{4}^{3}+15 a_{1}^{2} a_{2} a_{3}^{6} a_{4}^{4} \\
& -3 a_{1}^{2} a_{2}^{2} a_{3}^{8} a_{4}^{2}+3 a_{1}^{3} a_{2} a_{3}^{7} a_{4}^{3}-3 a_{3}^{2} a_{4}^{8}+a_{3}^{10} a_{4}^{2}+a_{3}^{6} a_{4}^{5}, \\
& D=15 a_{1} a_{2}^{2} a_{3}^{8} a_{4}^{2}-27 a_{1} a_{2} a_{3}^{6} a_{4}^{4}-18 a_{1}^{2} a_{2} a_{3}^{7} a_{4}^{3}+3 a_{1}^{2} a_{2}^{2} a_{3}^{9} a_{4}-3 a_{1}^{3} a_{2} a_{3}^{8} a_{4}^{2}+a_{2}^{2} a_{3}^{11}+4 a_{3}^{6} a_{4}^{7}-2 a_{3}^{11} a_{4} \\
& -a_{1} a_{2}^{3} a_{3}^{10}-3 a_{3}^{7} a_{4}^{4}-a_{1}^{2} a_{3}^{9} a_{4}^{2}+a_{1}^{4} a_{3}^{7} a_{4}^{3}+7 a_{1}^{3} a_{3}^{6} a_{4}^{4}-4 a_{2}^{3} a_{3}^{9} a_{4}+13 a_{1} a_{3}^{4} a_{4}^{6} \\
& -12 a_{2} a_{3}^{5} a_{4}^{5}+15 a_{1}^{2} a_{3}^{5} a_{4}^{5}+12 a_{2}^{2} a_{3}^{7} a_{4}^{3}+2 a_{2} a_{3}^{9} a_{4}^{2}-4 a_{1} a_{3}^{8} a_{4}^{3}, \\
& E=a_{1}^{2} a_{3}^{10} a_{4}-a_{1}^{3} a_{3}^{7} a_{4}^{3}-a_{1} a_{2} a_{3}^{11}-3 a_{1} a_{3}^{5} a_{4}^{5}+3 a_{2} a_{3}^{6} a_{4}^{4}-3 a_{1}^{2} a_{3}^{6} a_{4}^{4}-3 a_{2}^{2} a_{3}^{8} a_{4}^{2}-2 a_{2} a_{3}^{10} a_{4} \\
& +3 a_{1} a_{3}^{9} a_{4}^{2}+6 a_{1} a_{2} a_{3}^{7} a_{4}^{3}-3 a_{1} a_{2}^{2} a_{3}^{9} a_{4}+3 a_{1}^{2} a_{2} a_{3}^{8} a_{4}^{2}+2 a_{3}^{8} a_{4}^{3}-a_{3}^{4} a_{4}^{6}+a_{2}^{3} a_{3}^{10} \text {. }
\end{aligned}
$$

Thus first six term of $\left(h_{n}\right)$ are elements of the ring $R=\mathbb{Z}\left[a_{1}, a_{2}, a_{3}, a_{4}, x, y, h_{-1}^{ \pm 1}, h_{0}\right]$. By induction on $n>6$ that if $h_{n} \in R$, then $h_{n+1} \in$ $R$, which shows that each $h_{n}$ can be expressed as elements of the ring $R$.

In particular, the denominator of $h_{n}$ consists of only the term $h_{-1}$. Thus if $h_{-1}= \pm 1$, then $h_{n}$ $\in \mathbb{Z}$ since the values $a_{1}, a_{2}, a_{3}, a_{4}, x, y, h_{0}$ are integers.

From Lemma 3.1 we deduce that if $E$ is an elliptic curve given by equation (2.1) with integral coefficients and if $P, Q \in E$ are points as in Theorem 2.1, then the Somos 4 sequence $\left(h_{n}\right)$ with $h_{-1}= \pm 1$ consists entirely of integers for all $n \geq 0$, which completes the proof of main theorem.

\section{Linear Sequences and Somos 4 Sequences}

A linear recurrence sequence is a sequence $\left(h_{n}\right)$ which satisfies the recurrence relation $h_{n+k}=\alpha_{1} h_{n+k-1}+\alpha_{2} h_{n+k-2}+\ldots+\alpha_{k} h_{n}$

where the coefficients $\alpha_{1}, \ldots, \alpha_{k}$ and $k$ initial values $h_{0}, h_{1}, \ldots, h_{k-1}$ are given rationals.

The polynomial

$$
f(x)=x^{k}-\alpha_{1} x^{k-1}-\ldots-\alpha_{k-1} x-\alpha_{k}
$$

associated to relation (4.1) said to be its characteristic polynomial and the linear recurrence called order of $k$. For background and all properties of (linear) recurrence sequences we refer the reader (Everest et al, 2003). 
A linear sequence of order 2 (binary) always satisfies Somos 4 recurrence (1.2). For instance, the Fibonacci sequence $\left(s_{n}\right)$ defined by

$$
s_{n+1}=s_{n}+s_{n-1}
$$

is a Somos 4 sequence with coefficients

$$
\tau_{1}=-1, \quad \tau_{2}=2
$$

On the contrary a Somos 4 sequence $\left(h_{n}\right)$ with the following binary relation

$$
h_{n+1}=\alpha_{1} h_{n}+\alpha_{2} h_{n-1}
$$

$\left(\alpha_{1}, \alpha_{2} \in \mathbb{Q}\right)$ if the coefficients of $\left(h_{n}\right)$ are given by

$$
\tau_{1}=\frac{\left(h_{0} h_{3}-h_{1} h_{2}\right)^{2}}{\left(h_{1} h_{3}-h_{2}^{2}\right)\left(h_{0} h_{2}-h_{1}^{2}\right)}, \quad \tau_{2}=1-\tau_{1} .
$$

In this case, the constants $\alpha_{1}, \alpha_{2}$ are determined from the initial values $h_{0}, h_{1}, h_{2}$ and $h_{3}$ as follows:

$$
\alpha_{1}=\frac{h_{0} h_{3}-h_{1} h_{2}}{h_{0} h_{2}-h_{1}^{2}}, \quad \alpha_{2}=\frac{h_{2}^{2}-h_{1} h_{3}}{h_{0} h_{2}-h_{1}^{2}} .
$$

For more details see (Swart, 2003). In the following theorem we give the general terms of the Somos 4 sequences which satisfy a linear recurrence of order 2 .

Theorem 4.1. Let $\left(h_{n}\right)$ be a Somos 4 sequence with $\tau_{1}, \tau_{2} \neq 0$ and let $h_{0}, h_{1}, h_{2}$ and $h_{3}$ be its initial values. Suppose $\left(h_{n}\right)$ satisfy a binary linear recurrence relation. Then the general term of the sequence can be given by

$$
h_{n}=\varepsilon \alpha_{2}^{\lfloor n / 2\rfloor-1} h_{3}+\sum_{i=0}^{\lfloor n / 2\rfloor-2} \alpha_{1}^{n-(2 i+4)} \alpha_{2}^{i}\left\{\left(\begin{array}{c}
n-(i+3) \\
i
\end{array}\right) \alpha_{1} h_{3}+\left(\begin{array}{c}
n-(i+4) \\
i
\end{array}\right) \alpha_{2} h_{2}\right\}
$$

where

$$
\varepsilon= \begin{cases}0, & \text { if } n \text { is even } \\ 1, & \text { if } n \text { is odd }\end{cases}
$$

and $\alpha_{1}, \alpha_{2}$ as in (4.3).

Proof. We argue by induction on $n$. It is clear that the result is true for $n=4$. Suppose that $n>4$ and consider the case where $n$ is even. Then $n+1$ and $n-1$ are both odd. Now using the relations (4.2) we obtain

$$
\begin{gathered}
h_{n+1}=\alpha_{1} h_{n}+\alpha_{2} h_{n-1}=\alpha_{1}\left(\sum_{i=0}^{\lfloor n / 2\rfloor-2} \alpha_{1}^{n-(2 i+4)} \alpha_{2}^{i}\left\{\left(\begin{array}{c}
n-(i+3) \\
i
\end{array}\right) \alpha_{1} h_{3}+\left(\begin{array}{c}
n-(i+4) \\
i
\end{array}\right) \alpha_{2} h_{2}\right\}\right) \\
+\alpha_{2}\left(\sum_{i=0}^{\lfloor(n-1) / 2\rfloor-2} \alpha_{1}^{n-1-(2 i+4)} \alpha_{2}^{i}\left\{\left(\begin{array}{c}
n-1-(i+3) \\
i
\end{array}\right) \alpha_{1} h_{3}+\left(\begin{array}{c}
n-1-(i+4) \\
i
\end{array}\right) \alpha_{2} h_{2}\right\}\right) \\
=\alpha_{1}^{n-3}\left[\left(\begin{array}{c}
n-3 \\
0
\end{array}\right) \alpha_{1} h_{3}+\left(\begin{array}{c}
n-4 \\
0
\end{array}\right) \alpha_{2} h_{2}\right]+\alpha_{1}^{n-5} \alpha_{2}\left[\left(\begin{array}{c}
n-4 \\
1
\end{array}\right) \alpha_{1} h_{3}+\left(\begin{array}{c}
n-5 \\
1
\end{array}\right) \alpha_{2} h_{2}\right] \\
+\alpha_{1}^{n-7} \alpha_{2}^{2}\left[\left(\begin{array}{c}
n-5 \\
2
\end{array}\right) \alpha_{1} h_{3}+\left(\begin{array}{c}
n-6 \\
2
\end{array}\right) \alpha_{2} h_{2}\right]+\ldots
\end{gathered}
$$




$$
\begin{aligned}
& +\alpha_{1}^{3} \alpha_{2}^{\lfloor n / 2\rfloor-3}\left[\left(\begin{array}{c}
n-\lfloor n / 2\rfloor \\
\lfloor n / 2\rfloor-3
\end{array}\right) \alpha_{1} h_{3}+\left(\begin{array}{c}
n-\lfloor n / 2\rfloor-1 \\
\lfloor n / 2\rfloor-3
\end{array}\right) \alpha_{2} h_{2}\right] \\
& +\alpha_{1}^{n-5} \alpha_{2}\left[\left(\begin{array}{c}
n-4 \\
0
\end{array}\right) \alpha_{1} h_{3}+\left(\begin{array}{c}
n-5 \\
0
\end{array}\right) \alpha_{2} h_{2}\right]+\alpha_{1}^{n-7} \alpha_{2}^{2}\left[\left(\begin{array}{c}
n-5 \\
1
\end{array}\right) \alpha_{1} h_{3}+\left(\begin{array}{c}
n-6 \\
1
\end{array}\right) \alpha_{2} h_{2}\right] \\
& +\alpha_{1}^{n-9} \alpha_{2}^{3}\left[\left(\begin{array}{c}
n-6 \\
2
\end{array}\right) \alpha_{1} h_{3}+\left(\begin{array}{c}
n-7 \\
2
\end{array}\right) \alpha_{2} h_{2}\right]+\ldots \\
& +\alpha_{1} \alpha_{2}^{\lfloor n / 2\rfloor-2}\left[\left(\begin{array}{c}
n-\lfloor n / 2\rfloor-1 \\
\lfloor n / 2\rfloor-2
\end{array}\right) \alpha_{1} h_{3}+\left(\begin{array}{c}
n-\lfloor n / 2\rfloor-2 \\
n / 2\rfloor-2
\end{array}\right) \alpha_{2} h_{2}\right]+\alpha_{2}^{\lfloor n / 2\rfloor-1} h_{2} .
\end{aligned}
$$

Therefore we have

$$
\begin{aligned}
& h_{n+1}=\alpha_{1}^{n-3}\left[\left(\begin{array}{c}
n-2 \\
0
\end{array}\right) \alpha_{1} h_{3}+\left(\begin{array}{c}
n-3 \\
0
\end{array}\right) \alpha_{2} h_{2}\right]+\alpha_{1}^{n-5} \alpha_{2}\left[\left(\begin{array}{c}
n-3 \\
1
\end{array}\right) \alpha_{1} h_{3}+\left(\begin{array}{c}
n-4 \\
1
\end{array}\right) \alpha_{2} h_{2}\right] \\
& +\alpha_{1}^{n-7} \alpha_{2}^{2}\left[\left(\begin{array}{c}
n-4 \\
2
\end{array}\right) \alpha_{1} h_{3}+\left(\begin{array}{c}
n-5 \\
2
\end{array}\right) \alpha_{2} h_{2}\right]+\ldots \\
& +\alpha_{1} \alpha_{2}^{\lfloor n / 2\rfloor-2}\left[\left(\begin{array}{c}
n-\lfloor n / 2\rfloor-1 \\
\lfloor n / 2\rfloor-2
\end{array}\right) \alpha_{1} h_{3}+\left(\begin{array}{c}
n-\lfloor n / 2\rfloor-2 \\
\lfloor n / 2\rfloor-2
\end{array}\right) \alpha_{2} h_{2}\right]+\alpha_{2}^{\lfloor n / 2\rfloor-1} h_{2} \\
& =\sum_{i=0}^{\lfloor(n+1) / 2\rfloor-2} \alpha_{1}^{n+1-(2 i+4)} \alpha_{2}^{i}\left\{\left(\begin{array}{c}
n+1-(i+3) \\
i
\end{array}\right) \alpha_{1} h_{3}+\left(\begin{array}{c}
n+1-(i+4) \\
i
\end{array}\right) \alpha_{2} h_{2}\right\}+\alpha_{2}^{\lfloor(n+1) / 2\rfloor-1} h_{3}
\end{aligned}
$$

The case where $n$ is odd can be dealt with similarly.

\section{Discussions and Conclusions}

Teorem 2.2 provides an approach to determining Somos 4 sequences which have only integer terms. Our approach based on the relations in Theorem 2.1 and the elliptic equation (2.1) while (Hone and Swart, 2008), use the invariant (1.3). It would be interesting to use a similar method to obtain integrality properties of Somos 5 sequences. Finally, we derived a general term formula for Somos 4 sequences which satisfy a binary linear recurrence relation using the relations between these sequences. Thus the terms of such sequences can be easily calculated.

\section{References}

Everest, G., van der Poorten, A., Shparlinski, I. and Ward, T., (2003). "Recurrence Sequences", Mathematical Surveys and Monographs 104, AMS, Providence, RI; pp 318.

Fomin, S. and Zelevinsky, A. (2002). "The Laurent phenomen", Adv. Appl. Math., 28, 119-144.

Gale, D. (1991). "The strange and surprising saga of the Somos sequences", Math. Intelligencer, 13 (1) 40-42. 
Gale, D. (1991). "Somos sequence update", Math. Intelligencer, 13 (4) 49-50.

Gezer, B., Çapa, B. and Bizim, O. (2016), ”A family of integer Somos sequences", Mathematical Reports, 18 (68), 3 417435.

Hone, A. N. W. (2005), "Elliptic curves and quadratic recurrence sequences", Bull. Lond. Math. Soc., 37, 161--171; Corrigendum 38 (2006), 741-742.

Hone, A. N. W. and Swart, C. (2008). "Integrality and the Laurent phenomenon for Somos 4 and Somos 5 sequences", Math. Proc. of the Cambridge Phil. Soc., 145 65-85.

Husemöller, D. (1987). "Elliptic Curves", Springer Verlag, New York; pp 487.

Malouf, J. L. (1992). “An integer sequence from a rational recursion", Discrete Math., 110 257-261.

Propp, $\mathrm{J}$,

www.faculty.uml.edu/jpropp/somos.html, (accessed 09. 04.2018).

van der Poorten, A. J. (2005). "Elliptic curves and continued fractions", J. Int. Seq., 8, Article 05.2.5.

van der Poorten, A. J. (2006). "Hyperelliptic curves, continued fractions, and Somos sequences", IMS Lecture Notes Monograph Series. Dynamics \& Stochastics, 48 212-224.

Robinson, R. (1992). "Periodicity of Somos sequences", Proc. Amer. Math. Soc., 116 613-619.

Silverman, J. H. (2009). "The Arithmetic of Elliptic Curves" 2nd Edition, Graduate Texts in Mathematics 106, Springer Dordrecht Heidelberg London New York; pp 513.

Silverman, J. H. And Tate, J. (1992). "Rational Points on Elliptic Curves", Undergraduate Texts in Mathematics, Springer; pp 281

Swart, C. S. (2003). "Elliptic curves and related sequences", $\mathrm{Ph}$. D. thesis, Royal Holloway (University of London), pp 3-223.

Ward, M. (1948). "The law of repetition of primes in an elliptic divisibility sequences", Duke Math. J., 15 941946.

Ward, M. (1948). "Memoir on elliptic divisibility sequences", Amer. J. Math., 70

31-74. 